\title{
An Introduction to Robotically Assisted Surgical Systems: Current Developments and Focus Areas of Research
}

\author{
Julian Klodmann $^{1}$ (D) . Christopher Schlenk ${ }^{1}$ - Anja Hellings-Kuß ${ }^{1} \cdot$ Thomas Bahls $^{1} \cdot$ Roland Unterhinninghofen $^{1}$. \\ Alin Albu-Schäffer ${ }^{1}$. Gerd Hirzinger ${ }^{1}$
}

Accepted: 6 April 2021 / Published online: 26 August 2021

(C) The Author(s) 2021

\begin{abstract}
Purpose of Review Robotic assistance systems for diagnosis and therapy have become technically mature and widely available. Thus, they play an increasingly important role in patient care. This paper provides an overview of the general concepts of robotically assisted surgical systems, briefly revisiting historical and current developments in the surgical robotics market and discussing current focus areas of research. Comprehensiveness cannot be achieved in this format, but besides the general overview, references to further readings and more comprehensive reviews with regard to particular aspects are given. Therefore, the work at hand is considered as an introductory paper into the topic and especially addresses investigators, researchers, medical device manufacturers, and clinicians, who are new to this field.

Recent Findings The current research in Robotically Assisted Surgical Systems (RASS) increasingly uses established robotic platforms. To minimize the patient trauma while optimizing the dexterity of the surgeon, miniaturized instruments and semi-autonomous assistance functions are developed. To provide the surgeon with all necessary information in an adequate manner, novel imaging sensors as well as techniques for multimodal sensory feedback and augmented reality are investigated. The Surgical Data Science applies data management and processing approaches including machine learning on medical data to provide optimal, individualized and contextual support to the surgeon.
\end{abstract}

Summary Robotic systems will significantly influence future patient care. Since they must fulfill manifold medical, technical, regulatory and economic requirements, their development calls for a close, active and interdisciplinary cooperation between stakeholders from hospitals, industry and science.

Keywords Robotically assisted surgical systems $\cdot$ Surgical robotics

\section{Introduction}

Since the first conceptual ideas in the 1970s until today, more than 7 million procedures have been performed utilizing Robotically Assisted Surgical Systems (RASS). The Medical Robotics Database (MeRoDa), which was maintained until 2010 by the university clinics Mannheim and Heidelberg (Germany), listed over 450 medical robotics

This article is part of the Topical Collection on Medical and Surgical Robotics

Julian Klodmann

julian.klodmann@dlr.de

1 Institute of Robotics and Mechatronics, German Aerospace Center (DLR), Münchener Str. 20, 82234 Wessling, Germany projects worldwide. Only few of these research projects led to commercially available systems due to various reasons: Technological complexity, a difficult patent situation and regulatory barriers accompanied by absent standardization made the development of such systems high-risk and expensive [1]. Besides, the clinical relevance and the cost effectiveness for clinical utilization must be considered right from the beginning of the development [2]. Only this way a RASS can become a commercially successful product, which enhances the medical care for a large number of patients.

Generally, most RASS aim at a relief of the clinicians. As an example, positioning and holding robot arms relieve surgical assistants from permanent holding tasks and enable them to assist in other valuable tasks. Ergonomic and user-friendly system designs can contribute to a physical relief and a simplified instrument handling, which further 
enhances surgical precision and leads to cognitive relief. Besides these qualitative enhancements of the surgeon's general work, RASS can also enable gentler procedures, e.g. highly dexterous instrumentation enables smaller and less traumatic access into the body of the patient. From the clinics perspective, RASS have the potential to increase safety for clinicians and patients, to standardize the therapy quality even for differing capabilities of the clinicians and to reduce the hospitalization time and thereby the costs per patient while improving patient outcomes [3, 4].

Despite the challenges in developing RASS and establish them in the market, over 40 systems are already commercially available for various surgical applications and approximately the same amount is currently under development [1].

In this paper, a general overview of historical developments is given in "Historical Overview" and definitions and standards in robot assisted surgery are briefly introduced in "Definitions and Standards". The Section "Commercially Available RASS" discusses commercially available systems in different surgical domains and "Current Research Trends in RASS" describes some of the focus areas in current medical robotics research. The paper concludes in "Conclusion and Outlook" with current challenges in this dynamic field and encourages close, active and interdisciplinary cooperation between stakeholders from hospitals, industry and science to foster the further research and development of RASS.

\section{Historical Overview}

In the 1970s, a NASA concept study investigated the potential use of telemanipulation in robotic assistance systems for emergency medicine [5]. The proposed solution (see Fig. 1a) already integrated many features, which are characteristic for robot-assisted laparoscopy today: The surgeon watches the surgical site through the video image of an endoscopic camera and remotely controls the RASS at the patient-side via input devices from a surgeon console.

After the first treatment support system in arthroscopy, the Arthrobot, from the early 1980s [6], the first direct interventional support by a RASS on a human patient took place in 1985: A PUMA-200 industrial robot positioned and locked a biopsy channel during a computer tomography (CT)-guided brain biopsy in neurosurgery [7].

Almost simultaneously, the development of the ROBODOC ${ }^{\circledR}$ system for bone milling during the implantation of a total hip endoprosthesis began at the IBM T.J. Watson Center and U.C. Davis. The first clinical study followed in 1992 and in 1996 the system received the CE-certification for the European market [8]. Since 2014, the system is sold by THINK Surgical Inc. (Fremont, CA, USA). In 1992, the development of the Acrobot system (Active Constraint Robot), the first robotic assistance system in knee arthroplasty, was started at the Imperial College London [9]. Its technology forms the basis of the Mako system, which is currently sold by Stryker Corp. Medical (Kalamazoo, MI, USA).

In the field of minimally invasive surgery, the NASA concept was advanced by various research institutes from the mid-1980s onwards. During that time, the US military recognized the potential benefits of the technology for remote-controlled first aid for wounded soldiers and intensively promoted corresponding research. The companies Computer Motion Inc. and Intuitive Surgical Inc. were founded in 1989 and 1995 in Sunnyvale (CA, USA), continuing the research of the NASA and SRI (Stanford Research Institution) respectively for the commercial exploitation of the evolved technologies [10, 11]. In 1993, the AESOP arm of Computer Motion, the first system for positioning an endoscopic camera, received FDA (Food and Drug Administration) approval. In 2001, Computer Motion introduced the ZEUS system for minimally invasive surgery: This system had two arms with different instruments for manipulation tasks, one arm for holding the endoscope and

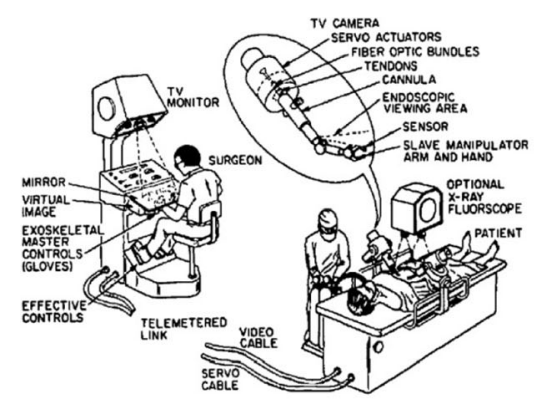

(a) Early Concept of a RASS (Reprinted by Permission from Springer Nature [5])

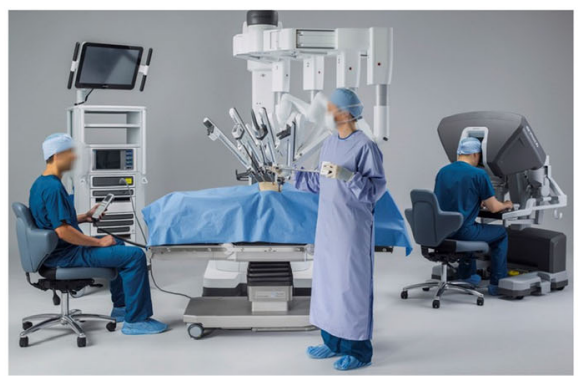

(b) Hillrom's TruSystem ${ }^{\circledR} 7000 \mathrm{dV}$ Surgical Table with the da Vinci Xi Surgical System (courtesy of Hillrom)

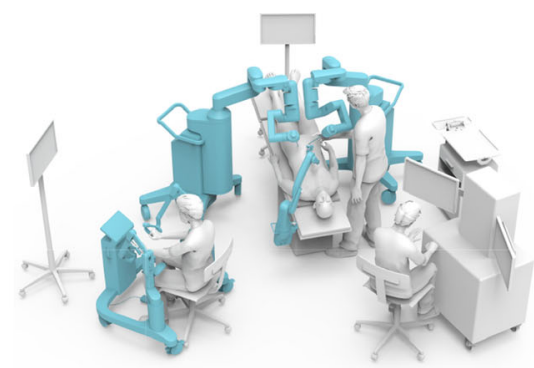

(c) Dexter System (courtesy of Distalmotion)

Fig. 1 Telemanipulation in robot assisted surgery 
was operated from a surgeon's console. In September 2001, a ZEUS system performed the first transatlantic gallbladder operation, in which a surgeon in New York operated on a patient in Strasbourg [12]. One year earlier, the FDA had approved Intuitive Surgical's da Vinci system. After the fusion of Intuitive Surgical and Computer Motion in 2003, the further development of AESOP and ZEUS was stopped and the systems were removed from the market. In Germany, the Forschungszentrum Karlsruhe developed in the early 1990s ARTEMIS, one the world's first demonstrators for minimally invasive robotic surgery. With a prototype of this teleoperation system for general and heart surgery, a laparoscopic cholecystectomy was successfully performed in a pig in 1996. However, the technology was not brought to market [13].

Further readings: $[2,6]$

\section{Definitions and Standards}

The lack of standards and definitions in surgical robotics leads to challenges in the further development of research prototypes into approved medical devices. Some manufacturers of surgical robots avoid denoting their systems as robots or integrating higher levels of automation to prevent the necessity of more lengthy and cost-intensive approval processes $[1,4]$. To facilitate these processes and at the same time ensure basic safety and essential performance of RASS, the standardization organizations ISO and IEC published the standard IEC/CD 80601-2-77 for surgical robots [14]. The ISO 8373:2012 (Robots and Robotic Devices Vocabulary) [15] defines robots as "[...] actuated mechanism programmable in two or more axes with a degree of autonomy, moving within its environment, to perform intended tasks".

This definition is further detailed by IEC/CD 80601-2-77 [14], which defines:

Robotically Assisted Surgical Equipment/System (RASE/RASS) "Medical Electrical Equipment/System that incorporates Programmable Electrical Medical System actuated mechanism intended to facilitate the placement or manipulation of Robotic Surgical Instrument."

Robotic Surgical Instrument (RSI) "Invasive device with applied part, intended to be manipulated by RASE or RASS to perform tasks in surgery."

Possibilities for further classification of a RASS by its degree of autonomy (DoA), are provided by the technical report IEC/TR 60601-4-1 [16]. The classification can in turn be used for risk analysis and finally in the application of the guidelines from IEC-CD 80601-2-77.

Further readings: [1, 4, 17]

\section{Commercially Available RASS}

The following subsections describe RASS for different surgical domains which are either already commercially available or announced to be available soon. The focus lies on systems from North America and Europe, since these systems are either already established or rather well documented.

\section{Laparoscopy}

Conventional laparoscopic surgery offers many advantages for the patient, in particular reduced trauma and shorter reconvalescence. However, there are significant disadvantages for the surgeon due to the ergonomics and handling of the instruments. The RASS for laparoscopic surgery are designed to reduce these challenges: The endoscope and the robotic instruments are carried by robotic arms. These instruments usually integrate two additional degrees of freedom (DoF) at the distal end compensating for the loss of two DoF at the incision point and a further functional DoF to actuate, e.g. gripper, forceps or needle holder. This allows the surgeon to remotely control, i.e. telemanipulate, the instruments in all six degrees of freedom using the input devices attached to an ergonomically designed surgeon console. Electrosurgery or other functions can be activated by foot pedals. Likewise, the foot pedals are used to couple the control between the input devices and the different instrument arms or the endoscope. The endoscope is usually a stereo endoscope that allows an enlarged 3D visualization of the surgical site. In most systems, hand-eye coordination is restored by matrix multiplication, such that the movement of the input device results in a rectified instrument movement in the endoscope image. To increase precision, human tremor can be filtered or the scaling between the surgeon's movement and the instrument tip can be adjusted. Together, these technical support functions are meant to facilitate surgical activities and, due to the resulting steeper learning curve, also enable novice surgeons to quickly perform minimally invasive interventions.

The most common RASS for laparoscopy is the da Vinci system from Intuitive Surgical (Sunnyvale, CA, USA). Currently the fourth generation, the da Vinci Xi System, is commercially available. Distinctive features of this system are the closed console concept and the attachment of all four robot arms to a common mount boom. Figure $1 \mathrm{~b}$ shows the da Vinci Xi System together with Hillrom's TruSystem $^{\circledR} 7000 d V$ surgical table, which is designed to work with integrated table motion so that a patient can be dynamically positioned while the surgeon operates. This allows the surgical team to efficiently manage the patient and utilize gravity for optimal exposure and access to the target anatomy. 
After the fusion with Computer Motion in 2003, Intuitive Surgical held a monopoly position in the field of minimally invasive robotic surgery for over ten years. One reason for this was the extensive patent portfolio, which Intuitive Surgical had built up over the years. Since many of the early patents are currently expiring, other companies now started developing their own RASS for minimally invasive surgery to market maturity. Obviously, the companies work on their own uniqueness in many aspects, e.g. system architecture, special features and economic aspects. From a technical perspective, especially the system architectures differ significantly in some aspects, such as the

- console design, e.g. open or rather closed console

- setup and mounting concepts of the robotic arms, e.g. boom-mounted, robots mounted on multiple carts, OR table mounted

- kinematics, e.g. remote center of motion kinematics or general serial kinematics.

With the Senhance ${ }^{\circledR}$ Surgical System from TransEnterix Surgical Inc. (Morrisville, NC, USA), the first system besides the da Vinci System was placed on the market in 2016 [18]. Each of its robot arms is attached to a boom on a separate cart and the surgeon console is open. Hand-eye coordination is not restored to replicate a similar handling as in conventional laparoscopic approaches. In 2019, the British company CMR Surgical Ltd. (Cambridge, UK) launched the Versius ${ }^{\circledR}$ Surgical Robotic System for laparoscopy [19]. It provides an open surgeon console and each kinematically redundant robot arm is mounted on its own cart. The spin-off company of the EPFL in Lausanne (Switzerland), Distalmotion SA (Epalinges, Switzerland) develops the Dexter robot with an open system architecture and an input console to be used in the sterile field (cmp. Fig. 1c, [20]). The robotic arms are mounted to booms on carts. Dexter is a three arm system composed of two instrument arms and one endoscope arm, all controlled fully robotically from the surgeon console. The open system architecture allows the use of any imaging system. An open surgeon console can be used in the sterile zone of the operating room, such that the surgeon can intraoperatively switch between laparoscopic and robotic surgery. The avatera System (Jena, Germany), the first commercial RASS developed in Germany, has received CE approval as a medical device for the European Economic Area [21]. Its four robotic arms are attached to a common mount boom, the surgeon teleoperates them from a closed console. Two of the world's largest medical device companies, Medtronic plc (Dublin, Ireland) and Johnson \& Johnson (New Brunswick, NJ, USA) have also announced RASS for laparoscopic soft tissue surgery. Medtronic's Hugo ${ }^{T M} R A S$ system is based on the DLR MiroSurge technology (cmp. Fig. 6,[22, 23]) and is expected to receive CE approval in 2021 [24]. The Johnson \& Johnson Ottava system is expected to enter clinical trials in 2022 and is developed by the company Verb Surgical (Santa Clara, CA, USA), founded in 2015 [25].

Further readings: [26, 27].

To reduce the patient trauma even further, one trend in medicine goes from traditional laparoscopic techniques, where several percutaneous incisions for the instruments and the camera are necessary, to Laparo-Endoscopic Single Site (LESS) (see [28]), where only one percutaneous incision is necessary. This operation technique requires strong miniaturization of instruments and endoscopes while at the same time each instrument needs two or more degrees of freedom to provide sufficient manipulation capabilities. With the SPORT system by Titan Medical Inc. (Toronto, Canada) [29] and the $d a$ Vinci $S P$ by Intuitive Surgical Inc.[30-32], first robotic systems for LESS are about to enter the market or are already commercially available, respectively.

\section{Spine and Neurosurgery}

In neurosurgery, various interventions can be supported by RASS, such as biopsies, placement of electrodes for deep brain stimulation or other cranial interventions. In spine surgery, the current main application of RASS is vertebral fixation (spondylodesis). In both surgical fields, the robotic systems serve as rigid guides or bearing devices for highprecision insertion of neurosurgical instruments, e.g. biopsy needles, drills or implants. A crucial step in this process is the intra-operative registration of the guiding device on the robot to the target structures on the patient and the preoperatively recorded imaging data, e.g. CT or MRI data. For this step, optical or electromagnetic navigation and tracking systems are typically used, which can continuously measure the spatial pose of marker targets [33]. However, laser scanners can also be used for a one-time static registration (cmp. [34]). Based on the registration, the preoperative plan is evaluated and-if necessary-adjusted. After the surgeon has verified the plan, the robotic system positions the tool guides, usually using the external navigation system to ensure the necessary precision.

Within the commercially available systems in this application area, two different system architectures can be distinguished (cmp. Fig. 2):

On the one hand, there are dedicated medical robots or adapted industrial robots with at least six degrees of freedom, which are equipped with a specialized guiding tool or end effector. Commercially available systems in these categories are, e.g. Mazor X Stealth ${ }^{T M}$ Edition System (Medtronic plc) [35], ROSA ONE ${ }^{\circledR}$ Spine/Brain System (Zimmer Biomet Holdings Inc.(Warsaw, IN, USA)) $[34,36]$, the ExcelsiusGPS ${ }^{\circledR}$ System (Globus Medical 
Fig. 2 a RASS in spine and neurosurgery assist in the stiff positioning of devices to guide neurosurgical tools with high precision; $\mathbf{b}$ and $\mathbf{c}$ RASS in orthopedics assist in precise bone removal for the insertion of joint endoprotheses

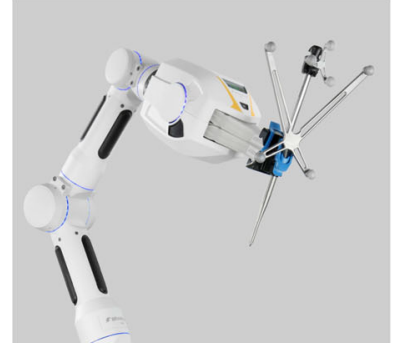

(a) Cirq ${ }^{\circledR}$ system (courtesy of Brainlab)

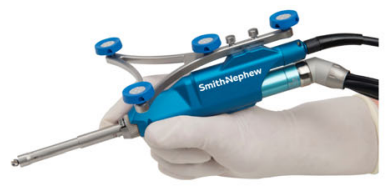

(b) Navio Surgical System (courtesy of Smith \& Nephew)

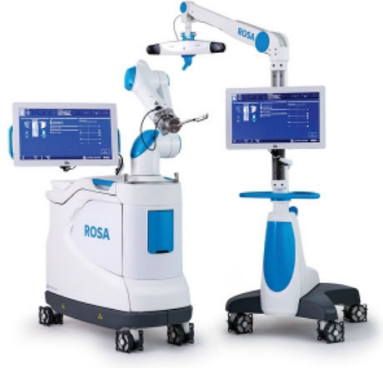

(c) ROSA ${ }^{\circledR}$ Knee System (courtesy of Zimmer Biomet)
Inc. (Audubon, PA, USA)) [37] and the neuromate ${ }^{\circledR}$ System (Renishaw plc (Gloucestershire, UK)) (cmp. [38]).

On the other hand, there are dedicated systems consisting of a passive, possibly partially weight-compensated holding arm for macro positioning and a small robotic end effector with high precision, which integrates the DoFs to meet the task space requirements. One example is the recently presented STEALTH AUTOGUIDE ${ }^{T M}$ System (Medtronic) for cranial neurosurgical interventions, whose development was originally initialized by the Austrian company iSYS Medizintechnik GmbH [39]. Another example is the Cirq ${ }^{\circledR}$ System of Brainlab (Munich, Germany) (cmp. Fig. 2a, [40]).

\section{Orthopedic Surgery}

In orthopedic surgery, RASS are used for joint reconstruction with knee and hip joint endoprostheses (cmp. Fig. 2b and c). Like in spine and neurosurgery, the implementation of the interventional plan is based on preoperatively acquired image data as well as an intraoperative registration between the robotically guided tools, the target structures on the patient and the preoperative data. For the intraoperative support of the surgeon there are different assistance concepts:

In a shared control approach, the surgeon manually guides the tool attached to the robot, e.g. an oscillating saw or milling machine. Initially, during coarse bone removal, the system supports visually. As soon as the tool reaches the margins of the bone to be removed, the system actively prevents the removal of bony structure beyond these margins. In Mako System (Stryker Corporation), the tool is attached to a robot arm. The robot control actively compensates for gravity, facilitating the manual guidance of the system. To keep within the margins, the robotic drives haptically render virtual walls, implemented, e.g. by active constraints or rather virtual fixture concepts [41, 42].

In the Navio Surgical System (Smith \& Nephew plc, London, UK), the robotic support of the handheld instruments during bone removal is implemented by an automatic cutting speed control and cutting stop autonomy (cmp. Fig. 2b, [43]).

The ROSA ${ }^{\circledR}$ Knee System (Zimmer Biomet Holdings Inc.) positions and fixes cutting blocks on the bone, similar to the insertion guide devices described in the previous section on spinal surgery. The surgeon moves the cutting tools manually along the guides (cmp. Fig. 2c, [44]).

The TSolution One ${ }^{\circledR}$ System (Think Surgical Inc.), a further development of the ROBODOC ${ }^{\circledR}$ system, autonomously performs the milling of the bone according to preoperative planning and intraoperative registration [45].

\section{Vascular Surgery}

In heart and vascular surgery, telemanipulated RASS are available for angioplasty interventions: They typically consist of a surgeon console, a holding arm with the actuation mechanism for the catheter cassette as end effector and a disposable catheter cassette. The actuation mechanism and the catheter cassette together allow a teleoperated motion of the catheter translationally and rotationally with respect to its main axis. Furthermore, the tip of the catheter can be steered to enable navigation through the vascular system. Once the guide wires, catheters and stents have been inserted, they can be remotely controlled with direct visualization from the radiation-protected ergonomic surgeon console. This allows a lower radiation exposure and a more ergonomic working position for the physicians, a higher precision in the placement of the stents and a reduction of vascular traumata. Although the current systems are purely telemanipulated, further navigation aids are conceivable, e.g. through complete integration of imaging angiography systems.

The French company Robocath Inc. (Rouen, France) is aiming for three fields of application with its $R$-One $e^{T M}$ system. Initially, percutaneous coronary interventions are supported. However, the system will also be enhanced for peripheral arterial diseases and mechanical thrombectomies [46]. The CorPath ${ }^{\circledR}$ GRX system (formerly Corindus Inc., 
since 2019 Siemens Healthcare GmbH, Erlangen, Germany) is also approved by the FDA for coronary and peripheral vascular interventions and has CE certification for the European market (cmp. Fig. 3, [47]).

\section{RASS for other Disciplines}

Also for many other diagnostic or interventional applications, e.g. in microsurgery, ophthalmology, otorhinolaryngology, radiation therapy or for endoluminal endoscopic procedures, such as bronchoscopy, different RASS are already commercially available or about to be launched. In the following some examples for RASS are given (cmp. "Definitions and Standards"):

Carl Zeiss Meditec AG (Jena, Germany) offers a selfdeveleoped robotic visualization system, the KINEVO ${ }^{\circledR} 900$, for neuro- and spinal surgery (cmp. Fig. 4a) [48]. In addition to the Corpath $^{\circledR} G R X$, the Siemens Healthcare GmbH also provide robotic angiography systems such as the ARTIS pheno ${ }^{\circledR}$ in their product portfolio [49] (cmp. Fig. 4b).

In general, the amount of the RASS relying on well established classical industrial robot technologies but also on collaborative or rather lightweight robots is increasing. KUKA for example adapts their robot systems for medical certification and application by their Medical Robotics division [50]. Particularly noteworthy is the KUKA LBR Med (cmp. Fig. 4c). It is certified according to the internationally recognized ECEE CB Scheme, i.e. it fulfills the international standards IEC 60601-1 and IEC 62304 [50]. This significantly simplifies the certification of RASS, which use this commercially available arm as robotic component. Thus, this robot is now a core component of numerous systems from various international companies, like, e.g. the AOT CARLO ${ }^{\circledR}$.

Further readings: $[1,26]$

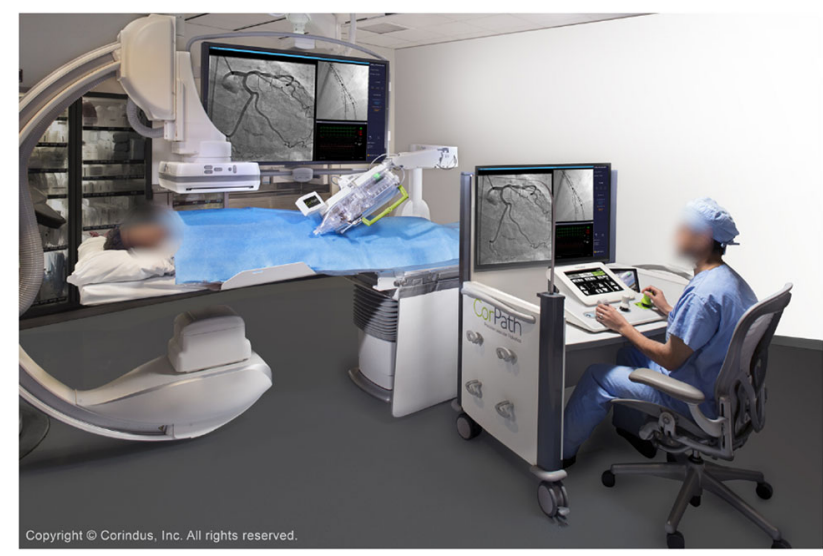

Fig. 3 RASS in vascular surgery like the CorPath ${ }^{\circledR}$ GRX can reduce radiation and improve the ergonomics for the surgeons as well as increase the precision of the catheter motion (courtesy of Corindus)

\section{Current Research Trends in RASS}

The current research in RASS is dynamic and manifold. Therefore, the subsequent section can only describe some of the most relevant trends but not provide a complete overview about the research activities in this field. Figure 5 depicts an overview of focus areas of research, which are referred to in the following paragraphs.

\section{Use of Established Robotic Platforms}

One current trend in RASS research is the use of established robotic platforms like the $d a$ Vinci Research Kit, the $R A V E N^{T M}$ or the KUKA LBR Med: The da Vinci Research Kit is an open-source research platform, which is based on the first generation of the da Vinci system. It was developed at Johns Hopkins University and is currently used at over thirty universities and research institutes in ten countries [51]. The system as a whole is described in [52], its software architecture in [53] and the dynamic model of its hand controllers and robotic arms in [54]. The RAVEN ${ }^{\mathrm{TM}}$, originally developed at the University of Washington, is a research platform for telesurgery in robot assisted laparoscopy and nowadays distributed by Applied Dexterity Inc. (Seattle, WA, USA) [55, 56]. The KUKA LBR Med, introduced in the previous section "RASS for other Disciplines", however, is an adaptation of the collaborative industrial lightweight robot $K U K A L B R$ iiwa for medical applications. Both the KUKA LBR Med and the lightweight robot for industry LBR iiwa base upon the lightweight robot technology of the Institute of Robotics and Mechatronics of DLR (cmp. Fig. 4c, [57]). Compared to self-developed research systems like the Hamlyn Arm [58] or the DLR MiroSurge [23], which is displayed in Fig. 6, those platforms provide less freedom regarding the hardware design and the control architecture. On the other hand, their hardware and software is well-tested, their broad availability simplifies cooperation with partners using the same system, and - at least in case of the KUKA LBR Med the transfer from research to a commercial product is facilitated.

\section{High Dexterity with Minimal Patient Trauma}

The schematic drawing in Fig. 5 visualizes the intraoperative relation between surgeon, robotic system and patient in robotic telesurgery. In such scenarios, the introduction of a RASS initially leads to a stronger physical separation between surgeon and patient: The surgeon does no longer directly interact with the patient but controls the system from the surgeon console. Consequently, the robotic system has to provide two channels between the surgeon and the patient: Firstly, it has to map the dexterity of the surgeon 
Fig. 4 Other examples of robotically assisted surgical systems

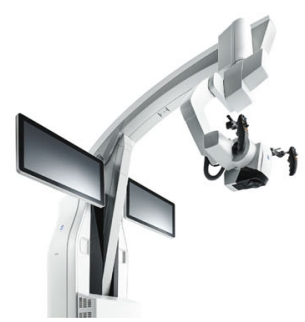

(a) $\mathrm{KINEVO}^{\circledR} 900$ (courtesy of Carl Zeiss Meditec)

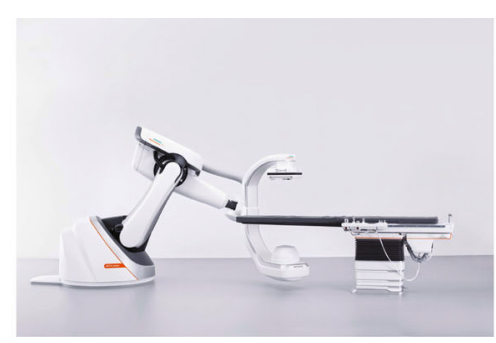

(b) ARTIS pheno ${ }^{\circledR}$ (courtesy of Siemens Healthcare)

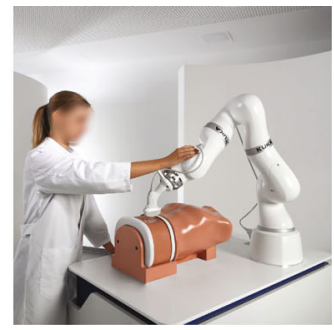

(c) LBR Med (courtesy of KUKA)

into the patient. Secondly, it has to provide the surgeon with all necessary information from the surgical site.

Projecting the surgeon's dexterity into the patient while at the same time keeping the trauma of the patient minimal, is a technically challenging goal, which motivates research in various fields:

- Design of miniaturized robotic instruments: Such instruments must be small in diameter to minimize the lesion of the patient, they must be stiff enough to allow for a sufficient manipulation force and they must provide sufficient motion capabilities to reach the surgical site and perform manipulation tasks there. To meet these competing requirements, a vast number of joint mechanisms has been developed (see, e.g. [59]). Especially for applications which require small instrument diameters and low manipulation forces, continuum robots become increasingly popular [60-62]. A critical aspect of this type of instrument is the compromise between manipulation force and flexibility.

- Less invasive operation techniques: The minimization of patient trauma remains a central goal of research in surgical robotics and has motivated the development of numerous robotic systems for LESS (cmp. "Laparoscopy", [63]). Alternative operation techniques

like Natural Orifice Transluminal Endoscopic Surgery (NOTES) (see [64]) and endoluminal interventions might reduce the patient trauma in the future even further: In NOTES, the surgical intervention is performed through the natural orifices of the human body (e.g. mouth, anus or vagina) and an incision in the luminar wall inside the body. In endoluminal interventions, the whole intervention takes place within a hollow organ of the human body (like, e.g. colon, stomach, lung or the urethral tract). First robotic systems for endoluminal interventions like the Flex ${ }^{\circledR}$ system by Medrobotics (Raynham, MA, USA) [62, 65] for otolaryngology and colorectal procedures and the Monarch ${ }^{\circledR}$ Platform by Auris Health Inc. (Redwood City, CA, USA) $[62,66]$ as well as the $\operatorname{Ion}^{T M}$ by Intuitive Surgical Inc., both targeting robot assisted bronchoscopy, have recently received FDA approval. One of the most challenging endoluminal interventions is colonoscopy, due to the complex shape of the human colon. This has triggered the technical improvement of flexible endoscopes (compare, e.g. [67]) as well as the development of novel robotic solutions. Overviews on Robotics in Colonoscopy and Gastrointestinal Endoscopy are provided in [68] and [69], respectively. Eventually, besides techniques for complex tissue manipulation through natural or

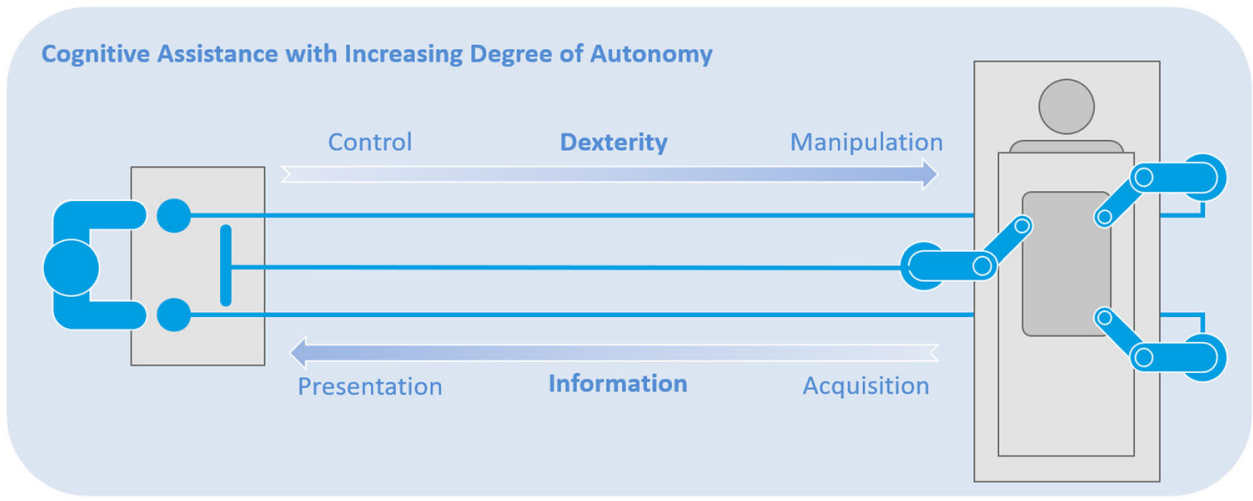

Fig. 5 Main research topics: acquisition and presentation of surgical site and patient information; control to project and enhance surgical dexterity into the patient and access hard-to-reach areas minimally invasive to manipulate tissue; augmentation of surgical skills through individualized and contextual assistance functions with reasonable degrees of autonomy for cognitive relief; seamless integration into the digitized surgical environment 
artificial incisions, robotically assisted solutions for percutaneous interventions, e.g. precise biopsies and needle based targeted therapies, gained attention in the community recently [70].

- Semi-autonomous assistance functions: Similar to the Mako ${ }^{\circledR}$ system in orthopedics, RASS in other fields might haptically guide the surgeon along resection lines or prevent unintended injury of sensitive structures by means of active constraints or rather virtual fixtures [41, 71-73]. Another approach to increase the degree of autonomy of RASS is the integration of instrument mechanisms into the robotic system that automate sub-tasks inherently mechanically, like, e.g. commercially available staplers, clip applicators or suturing instruments [1, 17, 74].

\section{Adequate Information for the Surgeon}

The goal of providing all necessary information in an adequate manner to the surgeon stimulates research in various fields:

- Multimodal sensory feedback: In commercially available systems for robot assisted laparoscopy and vascular surgery, transparent telepresence has not yet been achieved (cmp "Laparoscopy", "Vascular Surgery"). In both cases, there is a lack of haptic and tactile feedback, i.e. compensating for the surgeon's fingertip used in open surgery e.g. to classify tissue by palpation or in vascular surgery to palpate for bifurcations by tactile impressions when advancing a guide wire. While several studies indicated potential benefits of haptic feedback [75-77], the development of suitable costeffective, sterilizable or disposable miniaturized sensors for the integration into robotic instruments still remains a technological challenge [78-80]. However, some interesting concepts for a novel force-sensing laparoscopic instrument and the integration of force sensing capabilities into the trocar have been developed recently [81, 82].

- Novel imaging sensors and augmented reality: The intraoperative use of novel imaging sensors (e.g. integrating multi- or hyperspectral, ultrasound or photoacoustic imaging [83-85]) and the rapid development of machine learning, promotes research on various advanced imaging methods for the enrichment of surgical site information. While the intraoperative registration of bony structures is standard in orthopedics, it is still an unsolved challenge for deformable soft tissue structures such as in laparoscopy. This problem might be overcome in the future by means of annotated image data from numerous operations, advanced imaging and thereupon machine learning methods [86, 87]. As a result, relevant information taken from preoperative data, such as the location of vessels, nerves or tumors, could be presented to the surgeon using augmented- and mixed-reality solutions [88].

\section{Surgical Data Science}

Surgical Data Science generally deals with the collection, organization, analysis and modeling of medical data. While current RASS are still often encapsulated systems, there is a trend towards seamless integration of all technical systems in the OR, including the surgical robotic systems, into a common communication infrastructure. This poses different chances and technical challenges:

- Definition of communication standards for the OR: Currently, many manufacturers of medical devices use proprietary communication standards. The development of open standards and norms is a necessary condition for the common communication infrastructure described above. Early attempts like the IEEE 1073-1996 - IEEE Standard for Medical Device Communications, which defines interconnection and interoperation of medical devices, did not gain acceptance. As a consequence, numerous proprietary communication protocols complicate the data exchange across system borders and thus delay the efficient usage of data science in the surgical robotic domain.

- Definition of quality standards for medical data: Besides the different data formats and protocols, the lack of common quality standards also obstructs surgical data science. Varying skill levels of caretakers, different medical practices and diverse experience makes data difficult to compare [89]. Additionally, privacy and security of patient data have to be ensured [90].

- Modelling of surgical procedures: Another challenge is the description of surgical procedures in standardized

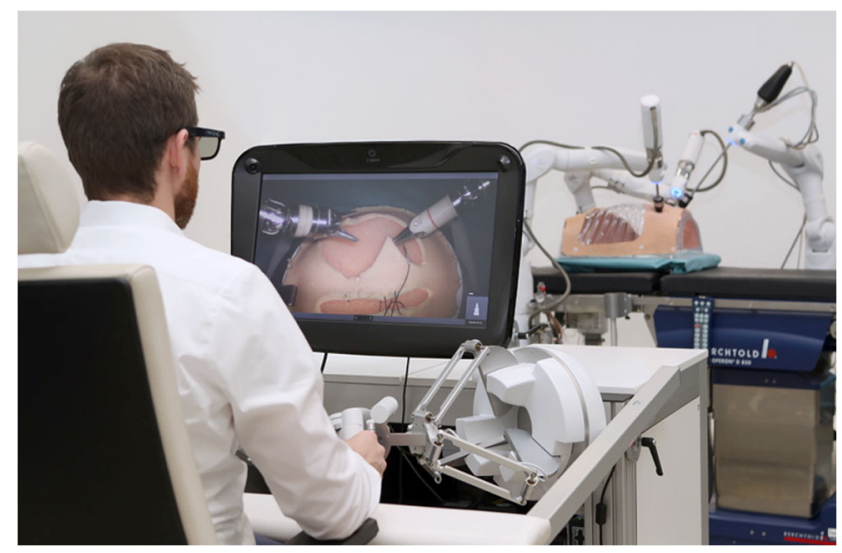

Fig. 6 MiroSurge-The DLR research platform for telemanipulation in minimally invasive robotic surgery (Alexandra Beier/DLR CC-BY 3.0) 
digital models [91-93]. This machine readable representation of knowledge, which is currently often only available in an implicit manner, is a crucial condition for increased autonomy of robotic systems as well as for context-aware assistance.

RASS could use procedural knowledge to provide optimal, individualized and contextual support to the surgeon and at the same time reduce the cognitive load [89, 94]. However, the success of the surgical data science strongly depends on the help of the clinicians. Only their support in the acquisition and annotation of data, i.e. the description of data segments with semantic medical information, can create the necessary data base for machine learning methods [95].

\section{Conclusion and Outlook}

The future of surgery will be significantly influenced by the use of RASS. Although there are major challenges in developing a prototype to market maturity, the relevant guidelines for basic safety and performance of these systems can be taken into account from the very beginning of the development, thus simplifying regulatory processes. In addition to these technical aspects, the clinical benefit and the cost for integration into the clinic must also be taken into account from the outset. Consequently, the successful development of a RASS can only be achieved through a close interdisciplinary partnership of scientists, (medical device) developers and clinicians. Bilateral, flexible adaptations and iterations of both the technology and the clinical procedure accelerate the implementation of novel solutions "from bench to bedside". This type of cooperation, the so-called Surgineering [96], requires an excellent mutual understanding of the disciplines, which should be promoted in the education of engineers and surgeons. Furthermore, RASS should be taken into account in the education of future surgeons in order to successfully manage the change in the operating room.

Close, active and interdisciplinary cooperation between surgeons and engineers in the development of new systems and the improvement of existing ones is essential to fully exploit the potential of RASS for patient care and thus for the well-being of the patient.

Funding Open Access funding enabled and organized by Projekt DEAL.

\section{Declarations}

Conflict of Interest The authors declare no competing interests.

Human and Animal Rights and Informed Consent This article does not contain any studies with human or animal subjects performed by any of the authors.
Open Access This article is licensed under a Creative Commons Attribution 4.0 International License, which permits use, sharing, adaptation, distribution and reproduction in any medium or format, as long as you give appropriate credit to the original author(s) and the source, provide a link to the Creative Commons licence, and indicate if changes were made. The images or other third party material in this article are included in the article's Creative Commons licence, unless indicated otherwise in a credit line to the material. If material is not included in the article's Creative Commons licence and your intended use is not permitted by statutory regulation or exceeds the permitted use, you will need to obtain permission directly from the copyright holder. To view a copy of this licence, visit http://creativecommons. org/licenses/by/4.0/.

\section{References}

1. Haidegger T. Autonomy for surgical robots: concepts and paradigms. IEEE Trans Med Robot Bionics. 2019;1(2):6576. Discussion of different aspects regarding autonomy of RASS, including an overview on current standardization activities.

2. Taylor RH, Menciassi A, Fichtinger G, Fiorini P, Dario P. Medical robotics and computer-integrated surgery. In: Springer handbook of robotics. Springer; 2016. p. 1657-84. General introduction of medical robotics and computer-integrated surgery as a part to a comprehensive textbook on robotics.

3. Porter ME, Larsson S, Lee TH. Standardizing patient outcomes measurement. N Engl J Med. 2016;374(6):504-06.

4. Yang GZ, Cambias J, Cleary K, Daimler E, Drake J, Dupont PE, et al. Medical robotics—regulatory, ethical, and legal considerations for increasing levels of autonomy. Sci Robot. 2017;2(4):8638.

5. Alexander AD. Impacts of telemation on modern society. 1973.

6. Takács A, Nagy DÁ, Rudas I, Haidegger T. Origins of surgical robotics: From space to the operating room. Acta Polytechnica Hungarica. 2016;13(1):13-30.

7. Kwoh YS, Hou J, Jonckheere EA, Hayati S. A robot with improved absolute positioning accuracy for CT guided stereotactic brain surgery. IEEE Trans Biomed Eng. 1988;35(2):153-60.

8. Kazanzides P, Zuhars J, Mittelstadt B, Williamson B, Cain P, Smith F, et al. Architecture of a surgical robot. In: Proceedings 1992 IEEE International Conference on Systems, Man, and Cybernetics. IEEE; 1992. p. 1624-29.

9. Davies B, Harris S, Lin W, Hibberd R, Middleton R, Cobb J. Active compliance in robotic surgery-the use of force control as a dynamic constraint. Proceedings of the Institution of Mechanical Engineers, Part H Journal of Engineering in Medicine. 1997;211(4):285-92.

10. NASA. Robotic surgery. 2000. Accessed: 12 Jun 2020. https:// spinoff.nasa.gov/spinoff2000/hm1.htm.

11. SRI International. Telerobotic surgery. 1995. Accessed: 12 Jun 2020. https://www.sri.com/hoi/telerobotic-surgery/.

12. Marescaux J, Leroy J, Gagner M, Rubino F, Mutter D, Vix M, et al. Transatlantic robot-assisted telesurgery. Nature. 2001;413(6854):379-80.

13. Schurr MO, Arezzo A, Buess GF. Robotics and systems technology for advanced endoscopic procedures: experiences in general surgery. Eur J Cardio-Thorac Surg. 1999;16(Supplement_2):S97_ S105.

14. IEC 80601-2-77:2019 Medical electrical equipment - Part 277: Particular requirements for the basic safety and essential performance of robotically assisted surgical equipment; 2019. Available from: https://www.iso.org/standard/68473.html. 
15. ISO 8373:2012 Robots and robotic devices - Vocabulary; 2012. Available from: https://www.iso.org/standard/55890.html.

16. IEC/TR 60601-4-1:2017 Medical electrical equipment - Part 41: Guidance and interpretation-medical electrical equipment and medical electrical systems employing a degree of autonomy; 2017. Available from: https://www.iso.org/standard/70755.html.

17. Attanasio A, Scaglioni B, De Momi E, Fiorini P, Valdastri P. Autonomy in surgical robotics. Annual Review of Control, Robotics, and Autonomous Systems. 2020;4.

18. TransEnterix Surgical, Inc. The Senhance ${ }^{\circledR}$ Surgical System with Digital Laparoscopy; 2020. Accessed: 2020-12-06. https:// transenterix.com/.

19. CMR Surgical Ltd. The Versius ${ }^{\circledR}$ Surgical Robotic SystemThe Versius ${ }^{\circledR}$ Surgical Robotic System; 2020. Accessed: 2020-12-06. https://cmrsurgical.com/versius.

20. Distalmotion SA. dexter - Just Surgery; 2020. Accessed: 2020-1206. https://www.distalmotion.com/product/.

21. avateramedical GmbH. avatera system; 2020. Accessed: 2020-1206. https://www.avatera.eu/en/avatera-system.

22. German Aerospace Center (DLR). DLR technology for robotassisted surgery; 2016. Accessed: 2020-12-06. https://www.dlr.de/ tm/en/desktopdefault.aspx/tabid-7986/14962_read-46838/.

23. Seibold U, Kübler B, Bahls T, Haslinger R, Steidle F. The DLR MiroSurge surgical robotic demonstrator. The Encyclopedia of Medical Robotics-Volume 1: Minimally Invasive Surgical Robotics. 2018;1:111-42.

24. Medtronic plc. Medtronic (2019) Robotic-assisted surgery(RAS) investor update.; 2019. Accessed: 2020-12-06. https://investorrelations.medtronic.com/events/event-details/robot ic-assisted-surgery-ras-investor-update.

25. Whooley S. Ottava surgical assistant robot finally unveiled by Johnson \& Johnson. Accessed: 2020-12-06. The Robot Report https://www.therobotreport.com/ottava-surgical-assistant- robotfinally-unveiled-by-johnson-johnson/. 2020.

26. Hoeckelmann M, Rudas IJ, Fiorini P, Kirchner F, Haidegger T. Current capabilities and development potential in surgical robotics. Int J Adv Robot Sys. 2015;12(5):61.

27. Rassweiler JJ, Autorino R, Klein J, Mottrie A, Goezen AS, Stolzenburg JU, et al. Future of robotic surgery in urology. BJU Int. 2017;120(6):822-41.

28. Autorino R, Kaouk JH, Stolzenburg JU, Gill IS, Mottrie A, Tewari A, et al. Current status and future directions of robotic single-site surgery: a systematic review. European Urology. 2013;63(2):266-80.

29. Seeliger B, Diana M, Ruurda JP, Konstantinidis KM, Marescaux J, Swanström LL. Enabling single-site laparoscopy: the SPORT platform. Surg Endosc. 2019;33(11):3696-703.

30. Agarwal DK, Sharma V, Toussi A, Viers BR, Tollefson MK, Gettman MT, et al. Initial experience with da vinci singleport robot-assisted radical prostatectomies. European Urology. 2020;77(3):373-79.

31. Dobbs RW, Halgrimson WR, Madueke I, Vigneswaran HT, Wilson JO, Crivellaro S. Single-port robot-assisted laparoscopic radical prostatectomy: initial experience and technique with the da Vinci® SP platform. BJU Int. 2019;124(6):1022-27.

32. Dobbs RW, Halgrimson WR, Talamini S, Vigneswaran HT, Wilson JO, Crivellaro S. Single-port robotic surgery: the next generation of minimally invasive urology. World $\mathrm{J}$ Urol. 2020;38(4):897-905.

33. Franz AM, Haidegger T, Birkfellner W, Cleary K, Peters TM, Maier-Hein L. Electromagnetic tracking in medicine-a review of technology, validation, and applications. IEEE Trans Med Imaging. 2014;33(8):1702-25.

34. Zimmer Biomet. ROSA ONE ${ }^{\circledR}$ Brain; 2020. Accessed: 2020-1206. https://www.zimmerbiomet.com/medical-professionals/cmf/ rosa-brain.html.
35. Medtronic plc. MAZOR X STEALTH EDITION ${ }^{\mathrm{TM}}$ Robotic Guidance System for Spinal Surgery; 2020. Accessed: 2020-12-06. https://www.medtronic.com/us-en/health care-professionals/products/spinal-orthopaedic/spine-robotics/ mazor-X-stealth-edition.html.

36. Zimmer Biomet. ROSA ONE ${ }^{\circledR}$ Spine; 2020. Accessed: 202012-06. https://www.zimmerbiomet.com/medical-professionals/ robotic-solutions/rosa-spine.html.

37. Globus Medical Inc. ExcelsiusGPS ${ }^{\circledR} ; 2020$. Accessed: 2020-1206. https://www.globusmedical.com/musculoskeletal-solutions/ excelsiusgps/.

38. Renishaw plc. neuromate ${ }^{\circledR}$ robotic system for stereotactic neurosurgery; 2020. Accessed: 2020-12-06. https://www.renishaw. com/en/neuromate-robotic-system-for-stereotactic-neurosurgery10712.

39. Medtronic plc. STEALTH AUTOGUIDE ${ }^{\mathrm{TM}}$ - Cranial Robotic Guidance Platform; 2020. Accessed: 2020-12-06. https://www. medtronic.com/us-en/healthcare-professionals/products/neurologi $\mathrm{cal} /$ cranial-robotics/stealth-autoguide.html.

40. Brainlab AG. Brainlab acquires robotics platform company Medineering; 2019. Accessed: 2020-12-06. https://www.brainlab.com/ de/press-releases/brainlab-acquires-robotics-platform-companymedineering/.

41. Bowyer SA, Davies BL. Rodriguez y Baena F. Active constraints/virtual fixtures: a survey. IEEE Trans Robot. 2013;30(1):138-57.

42. Hagag B, Abovitz R, Kang H, Schmitz B, Conditt M. RIO: Robotic-Arm Interactive Orthopedic system MAKOplasty: User interactive haptic orthopedic robotics. In: Surgical robotics. Springer; 2011. p. 219-46.

43. Smith \& Newphew plc. NAVIO Handheld Robotics; 2020. Accessed: 2020-12-06. https://www.smith-nephew.com/professional/ microsites/navio/navio-technology/product-overview/.

44. Zimmer Biomet. ROSA ${ }^{\circledR}$ Knee System; 2020. Accessed: 202012-06. https://www.zimmerbiomet.com/medical-professionals/ knee/product/rosa-knee.html.html.

45. THINK Surgical ${ }^{\circledR}$ Inc. Active Robot Technology; 2020. Accessed: 2020-12-06. https://thinksurgical.com/professionals/technology/.

46. Robocath Inc. The medical robot R-One; 2020. Accessed: 202012-06. https://www.robocath.com/product/.

47. Siemens Healthcare GmbH. CorPath GRX - Precision Vascular Robotics; 2020. Accessed: 2020-12-06. https://www.siemenshealthineers.com/en-us/angio/endovascular-robotics/precisionvascular-robotics.

48. Carl Zeiss Meditec AG. ZEISS KINEVO 900 - Redefining surgical visualization for advanced surgical certainty; 2020. Accessed: 2020-12-06. https://www.zeiss.com/meditec/us/ products/neurosurgery/surgical-microscopes/kinevo-900.html.

49. Siemens Healthcare GmbH. ARTIS pheno - As individual as your patients; 2020. Accessed: 2020-12-06. https://www.siemenshealthineers.com/de/angio/artis-interventional-angiographysystems/artis-pheno.

50. KUKA Deutschland GmbH, Medical Robotics. KUKA Healthcare; 2020. Accessed: 2020-12-06. https://www.kuka.com/en-de/ industries/health-care.

51. Intuitive Foundation. The da Vinci Research Kit; 2020. Accessed: 2020-12-15. http://www.intuitive-foundation.org/dvrk/.

52. Kazanzides P, Chen Z, Deguet A, Fischer GS, Taylor RH, DiMaio SP. An open-source research kit for the da Vinci ${ }^{\circledR}$ Surgical System. In: 2014 IEEE international conference on robotics and automation (ICRA). IEEE; 2014. p. 6434-39.

53. Chen Z, Deguet A, Taylor RH, Kazanzides P. Software architecture of the da Vinci Research Kit. In: 2017 First IEEE International Conference on Robotic Computing (IRC). IEEE; 2017. p. 180-87.

54. Fontanelli GA, Ficuciello F, Villani L, Siciliano B. Modelling and identification of the da Vinci research kit robotic arms. In: 
2017 IEEE/RSJ International Conference on Intelligent Robots and Systems (IROS). IEEE; 2017. p. 1464-69.

55. Hannaford B, Rosen J, Friedman DW, King H, Roan P, Cheng L, et al. Raven-II: an open platform for surgical robotics research. IEEE Trans Biomed Eng. 2012;60(4):954-59.

56. Applied Dexterity. RAVEN ${ }^{\mathrm{TM}}$; 2020. Accessed: 2020-12-06. https://applieddexterity.com/.

57. German Aerospace Center (DLR). History of the DLR LWR; 2004. Accessed: 2020-12-06. https://www.dlr.de/rm/en/ desktopdefault.aspx/tabid-12464/21732_read-44586/.

58. Wisanuvej P, Leibrandt K, Liu J, Yang GZ. Hands-on reconfigurable robotic surgical instrument holder arm. In: 2016 IEEE/RSJ International Conference on Intelligent Robots and Systems (IROS). IEEE; 2016. p. 2471-76.

59. Jelínek F, Arkenbout EA, Henselmans PW, Pessers R, Breedveld P. Classification of joints used in steerable instruments for minimally invasive surgery - a review of the state of the art. Journal of Medical Devices. 2015;9(1).

60. Burgner-Kahrs J, Rucker DC, Choset H. Continuum robots for medical applications: a survey. IEEE Trans Robot. 2015;31(6):126180.

61. Bergeles C, Yang GZ. From passive tool holders to microsurgeons: safer, smaller, smarter surgical robots. IEEE Trans Biomed Eng. 2013;61(5):1565-76.

62. Omisore OM, Han S, Xiong J, Li H, Li Z, Wang L. A review on flexible robotic systems for minimally invasive surgery. IEEE Transactions on Systems, Man, and Cybernetics: Systems. 2020; Review of robotic systems for minimally invasive surgery with multiple degrees of freedom.

63. Peters BS, Armijo PR, Krause C, Choudhury SA, Oleynikov D. Review of emerging surgical robotic technology. Surg Endosc. 2018;32(4):1636-55.

64. Halim I, Tavakkolizadeh A. The next surgical revolution? Int J Surg (London England). 2008;6(4):273.

65. Lang S, Mattheis S, Hasskamp P, Lawson G, Güldner C, Mandapathil M, et al. A European multicenter study evaluating the flex robotic system in transoral robotic surgery. The Laryngoscope. 2017;127(2):391-95.

66. Graetzel CF, Sheehy A, Noonan DP. Robotic bronchoscopy drive mode of the Auris Monarch platform. In: 2019 International Conference on Robotics and Automation (ICRA). IEEE; 2019. p. 3895-01.

67. Loeve A, Breedveld P, Dankelman J. Scopes too flexible... and too stiff. IEEE Pulse. 2010;1(3):26-41.

68. Ciuti G, Skonieczna-Żydecka K, Marlicz W, Iacovacci V, Liu H, Stoyanov D, et al. Frontiers of robotic colonoscopy: A comprehensive review of robotic colonoscopes and technologies. J Clinical Med. 2020;9(6):1648. Overview of RASS for colonoscopy.

69. Boškoski I, Orlandini B, Papparella LG, Matteo MV, De Siena $\mathrm{M}$, Pontecorvi $\mathrm{V}$, et al. Robotics and artificial intelligence in gastrointestinal endoscopy: updated review of the literature and state of the art. Current Robotics Reports. 2021;1-12.

70. Siepel FJ, Maris B, Welleweerd MK, Groenhuis V, Fiorini P, Stramigioli S. Needle and biopsy robots: a review. Current Robotics Reports. 2021;1-12.

71. Li Z, Gordon A, Looi T, Drake J, Forrest C, Taylor RH. Anatomical mesh-based virtual fixtures for surgical robots. In: 2020 IEEE/RSJ International Conference on Intelligent Robots and Systems (IROS); 2020. p. 3267-73.

72. Marinho MM, Adorno BV, Harada K, Mitsuishi M. Active constraints using vector field inequalities for surgical robots. In: 2018 IEEE International Conference on Robotics and Automation (ICRA). IEEE; 2018. p. 5364-71.
73. Marinho MM, Adorno BV, Harada K, Mitsuishi M. Dynamic active constraints for surgical robots using vectorfield inequalities. IEEE Trans Robot. 2019;35(5):116685.

74. Shademan A, Decker RS, Opfermann JD, Leonard S, Krieger A, Kim PC. Supervised autonomous robotic soft tissue surgery. Sci Transl Med. 2016;8(337):337ra64-64.

75. Enayati N, De Momi E, Ferrigno G. Haptics in robotassisted surgery: Challenges and benefits. IEEE Rev Biomed Eng. 2016;9:49-65.

76. Weber B, Eichberger C. The benefits of haptic feedback in telesurgery and other teleoperation systems: a meta-analysis. In: International conference on universal access in human-computer interaction. Springer; 2015. p. 394-405.

77. Miller J, Braun M, Bilz J, Matich S, Neupert C, Kunert W, et al. Impact of haptic feedback on applied intracorporeal forces using a novel surgical robotic system-a randomized cross-over study with novices in an experimental setup. Surgical Endoscopy. 2020;1-10.

78. Westebring-van der Putten EP, Goossens RH, Jakimowicz JJ, Dankelman J. Haptics in minimally invasive surgery-a review. Minim Invasiv Ther Allied Technol. 2008;17(1):3-16.

79. Haslinger R, Leyendecker P, Seibold U. A fiberoptic forcetorque-sensor for minimally invasive robotic surgery. In: 2013 IEEE international conference on robotics and automation. IEEE; 2013. p. 4390-95.

80. Leyendecker P, Haslinger R. Fiber optic curvature sensor. In: SENSORS, 2014 IEEE. IEEE; 2014. p. 43-46.

81. Liu H, Selvaggio M, Ferrentino P, Moccia R, Pirozzi S, Bracale U, et al. The MUSHA Hand II: a multi-functional hand for robot-assisted laparoscopic surgery. IEEE/ASME Transactions on Mechatronics. 2020.

82. Fontanelli GA, Buonocore LR, Ficuciello F, Villani L, Siciliano B. A novel force sensing integrated into the trocar for minimally invasive robotic surgery. In: 2017 IEEE/RSJ International Conference on Intelligent Robots and Systems (IROS). IEEE; 2017. p. 131-36.

83. Shapey J, Xie Y, Nabavi E, Bradford R, Saeed SR, Ourselin $\mathrm{S}$, et al. Intraoperative multispectral and hyperspectral labelfree imaging: a systematic review of in vivo clinical studies. J Biophotonics. 2019;12(9):e201800455.

84. Attia ABE, Balasundaram G, Moothanchery M, Dinish U, Bi R, Ntziachristos $\mathrm{V}$, et al. A review of clinical photoacoustic imaging: Current and future trends. Photoacoustics. 2019;16:100144.

85. von Haxthausen F, Böttger S, Wulff D, Hagenah J, GarcíaVázquez V, Ipsen S. Medical robotics for ultrasound imaging: current systems and future trends. Current Robotics Reports. 2021;1-17.

86. Esteva A, Robicquet A, Ramsundar B, Kuleshov V, DePristo M, Chou K, et al. A guide to deep learning in healthcare. Nat Med. 2019;25(1):24-29.

87. Zhou SK, Greenspan H, Davatzikos C, Duncan JS, van Ginneken B, Madabhushi A, et al. A review of deep learning in medical imaging: imaging traits, technology trends, case studies with progress highlights, and future promises. Proceedings of the IEEE. 2021.

88. Qian L, Wu JY, DiMaio SP, Navab N, Kazanzides P. A review of augmented reality in robotic-assisted surgery. IEEE Trans Med Robot Bionics. 2019;2(1):1-16.

89. Maier-Hein L, Vedula SS, Speidel S, Navab N, Kikinis R, Park A, et al. Surgical data science for next-generation interventions. Nat Biomed Eng. 2017;1(9):691-96. Introduction of Surgical Data Science as a new field of research for the future surgical care of patients. 
90. Kaur P, Sharma M, Mittal M. Big data and machine learning based secure healthcare framework. Procedia Comput Sci. 2018;132:1049-59.

91. Lalys F, Jannin P. Surgical process modelling: a review. Int J Comput Assist Radiol Surg. 2014;9(3):495-511.

92. Neumuth T, Jannin P, Strauss G, Meixensberger J, Burgert O. Validation of knowledge acquisition for surgical process models. J Am Med Inf Assoc. 2009;16(1):72-80.

93. Gibaud B, Forestier G, Feldmann C, Ferrigno G, Gonçalves P, Haidegger $\mathrm{T}$, et al. Toward a standard ontology of surgical process models. Int J Comput Assist Radiol Surg. 2018;13(9):1397-408.
94. Maier-Hein L, Eisenmann M, Sarikaya D, März K, Collins T, Malpani A, et al. Surgical data science-from concepts to clinical translation. arXiv:2011.02284. 2020.

95. Warren E. Strengthening research through data sharing. New England J Med. 2016;375(5):401-03.

96. Feussner H, Wilhelm D, Navab N, Knoll A, Lüth T. Surgineering: a new type of collaboration among surgeons and engineers. Int J Comput Assist Radiol Surg. 2019;14:187-90.

Publisher's Note Springer Nature remains neutral with regard to jurisdictional claims in published maps and institutional affiliations. 